This item was submitted to Loughborough's Research Repository by the author.

Items in Figshare are protected by copyright, with all rights reserved, unless otherwise indicated.

\title{
Noncontact blood perfusion mapping in clinical applications
}

PLEASE CITE THE PUBLISHED VERSION

http://dx.doi.org/10.1117/12.2225216

PUBLISHER

(c) SPIE

VERSION

VoR (Version of Record)

LICENCE

CC BY-NC-ND 4.0

REPOSITORY RECORD

lakovlev, Dmitry, Vincent M. Dwyer, Sijung Hu, and Vadim V. Silberschmidt. 2019. "Noncontact Blood Perfusion Mapping in Clinical Applications". figshare. https://hdl.handle.net/2134/23661. 


\title{
Noncontact blood perfusion mapping in clinical applications
}

\author{
Dmitry Iakovlev ${ }^{\mathrm{a}}$, Vincent Dwyer ${ }^{\mathrm{a}}$, Sijung $\mathrm{Hu}^{*^{\mathrm{a}}}$, Vadim Silberschmidt ${ }^{\mathrm{b}}$ \\ ${ }^{a}$ Photonics Engineering and Health Technology Research Group; ${ }^{b}$ Mechanics of Advanced Materials \\ and Modern Processes, Wolfson School, Loughborough University, Loughborough, LE11 3TU, UK
}

\begin{abstract}
Non-contact imaging photoplethysmography (iPPG) to detect pulsatile blood microcirculation in tissue has been selected as a successor to low spatial resolution and slow scanning blood perfusion techniques currently employed by clinicians. The proposed iPPG system employs a novel illumination source constructed of multiple high power LEDs with narrow spectral emission, which are temporally modulated and synchronised with a high performance sCMOS sensor. To ensure spectrum stability and prevent thermal wavelength drift due to junction temperature variations, each LED features a custom-designed thermal management system to effectively dissipate generated heat and auto-adjust current flow. The use of a multi-wavelength approach has resulted in simultaneous microvascular perfusion monitoring at various tissue depths, which is an added benefit for specific clinical applications. A synchronous detection algorithm to extract weak photoplethysmographic pulse-waveforms demonstrated robustness and high efficiency when applied to even small regions of $5 \mathrm{~mm}^{2}$. The experimental results showed evidences that the proposed system could achieve noticeable accuracy in blood perfusion monitoring by creating complex amplitude and phase maps for the tissue under examination.
\end{abstract}

Keywords: Imaging photoplethysmography, blood perfusion, light-tissue interaction, synchronous detection, biomedical assessment, multi-wavelength illumination, noncontact

\section{INTRODUCTION}

Blood perfusion represents a blood circulation through tissue capillary and vessel networks associated with transportation of oxygen, nutrients and waste products in body. Alternation in perfusion can serve as a criterion of underlying negative cellular metabolism including burns, wounds, ischemia and tumour tissue growth ${ }^{[1]}$. Hence, the demand to rapidly verify and characterise blood perfusion has been expanding.

\subsection{Conventional PPG}

Photoplethysmography (PPG), an optical technique for non-invasive measurement of blood volume changes, has found clinical use in pulse oximeters, which monitor peripheral blood oxygen saturation $\left(\mathrm{SpO}_{2}\right)$ and heart rate. The core of this technology is a complex nature of the light-tissue interaction, where light is absorbed, scattered, reflected and transmitted while penetrating through tissues ${ }^{[2]}$. Light, usually from a 500-1000 nm diode, is incident to the tissue surface. If the blood volume change is present in the microvascular bed of tissue due to contraction and expansion of blood vessels during a cardiac cycle, the amount of light being backscattered and absorbed varies. A photodetector located on skin surface measures small variations in light intensity arising from this light-tissue interaction, resulting in a dynamic cardiovascular pulse wave.

However, the contact PPG technology is limited to monitoring a single site with a volume of few cubic centimetres, as well as need to physically attach the probe to tissue surface, which could restrict its potential utilisation in some application, e.g. in open wound diagnostic.

\subsection{Imaging PPG}

Non-contact imaging photoplethysmography (iPPG) introduced a transition from a single-point measurement of light intensity variations into a large tissue area monitoring. iPPG is further development of conventional contact photoplethysmography, where a single photodetector is replaced by a digital photosensor array. With iPPG a simultaneous inspection of multiple tissue sites and detection of local blood perfusion variations is possible. Additionally, removal of wires and contact probes opens new diagnostic opportunities, e.g. placing virtual sensors on organs mucosa or wounds ${ }^{[3]}$.

*s.hu@lboro.ac.uk; phone +44 (0)1509 227058;

Biophotonics: Photonic Solutions for Better Health Care V, edited by Jürgen Popp, Valery V. Tuchin,

Dennis L. Matthews, Francesco Saverio Pavone, Proc. of SPIE Vol. 9887, 988712

(c) 2016 SPIE · CCC code: 0277-786X/16/\$18 - doi: 10.1117/12.2225216

Proc. of SPIE Vol. $9887988712-1$ 
iPPG has been an area of intensive research for the past decade focusing on areas such as feasibility study, motion compensation and signal processing. Our previous work demonstrated that this technology offers significant advantage over other blood perfusion scanning techniques, since there are evidences showing a viability of a low-cost iPPG system utilising a conventional web-camera ${ }^{[4]}$. Simultaneous monitoring of perfusion at different tissue depth using dualwavelength light source was also reported ${ }^{[5]}$, which could lead to the potential of non-contact 3D mapping of microcirculation. To date, however, iPPG has not found major appreciation in certified medical applications, despite numerous literature reviews of its clinical value and validity.

\subsection{Inspiration for this research project}

Since the quality of iPPG results depends on complex light-tissue interactions, the ability to precisely control a light source in repetitive manner is vital. The effects of variations in instrumentation had not been fully evaluated, thus making iPPG system behaviour less certain and its potential applications less attractive to the medical community. The primary aim of this research is to investigate an effective way to obtain reliable blood perfusion results by proposing a simple yet effective multi-wavelength light source and means of monitoring and managing its spectral behaviour over time. A synchronous detection method to de-noise iPPG signals and compare microvascular bed condition at various tissue sites and depths was evaluated and complex amplitude and phase results for the site under examination were obtained.

\section{METHODS}

\subsection{Multi-wavelength iPPG system}

The practical depth, at which an iPPG system can operate reliably, depends on the wavelength's ability to penetrate given tissue. There is no absolute formula for calculating this depth as this parameter is not only spectrum specific, but is also dependent on the tissue composition in the region of interest. Empirical studies conducted in-vitro and in-vivo and complex modelling using tissue-specific absorption and scattering coefficients suggested that in the visible spectrum higher wavelength light penetrates deeper into human skin ${ }^{[6]}{ }^{[7]}{ }^{[8]}$. In this experiment a multi-wavelength light source with light emitters at 530, 590 and $660 \mathrm{~nm}$ was selected to illuminate a palm area. Thus, by selecting certain wavelengths, iPPG system could achieve simultaneous monitoring of perfusion and microcirculation at desired tissue depths.

\subsection{Light source}

Since the amplitude of pulsatile blood (AC component) and quasi-static (DC component) are directly proportional to the intensity of backscattered light, it is beneficial to maximise light source optical power output so the sensor explores its maximum dynamic range without saturating. In order to create such evenly distributed light flux at distances greater than $50 \mathrm{~cm}$ from a target area, the illuminator is usually constructed using an array of individual LEDs, which has been reported in our previous work ${ }^{[5]}{ }^{[9]}$, as well as in ${ }^{[10]}$. One obvious yet underestimated disadvantage of this approach is inevitable spectral variation between individual LEDs even within the same production batch, with a typical value of around 20-40 nm. As a result, the combined array of multiple LEDs could produce illumination of wide bandwidth and uneven distribution. Since the light penetration depth is a function of light wavelength for a given tissue region, the spectral stability and narrow bandwidth are critical factors for applications where repeatability and reproducibility are required, such as pathology monitoring in epidermis and dermis. Wavelength variation between LEDs can be resolved by selecting components with similar spectral behaviour (also known as binning), but it requires additional verification stage and is only feasible at mass production.

In order to overcome these challenges and simplify the overall design, a novel single light source assembly with one high-power LED element per wavelength and a single collimating lens has been proposed (Figure 1). Compared to an array, spectral behaviour of a single LED is easier to inspect and verify with a spectrometer and an optical power meter prior to its commissioning. In the event of performance deviation over time, this element can be effectively replaced without affecting the light source assembly.

As shown in Figure 1, the proposed light source consists of three high-power LEDs (LXZ1 Series, Philips, USA) at 530, 590 and $660 \mathrm{~nm}$ with typical half-power bandwidth of $20 \mathrm{~nm}$. Spectral properties were quantified using a calibrated spectrometer (USB4000, Ocean Optics, USA) and an optical power meter (Model 835, Newport, USA), which were synchronised with the light source to record parameters during image acquisition. This approach allows monitoring of 
light source spectral behaviour over time and under various LED forward currents for greater experiment control and repeatability.

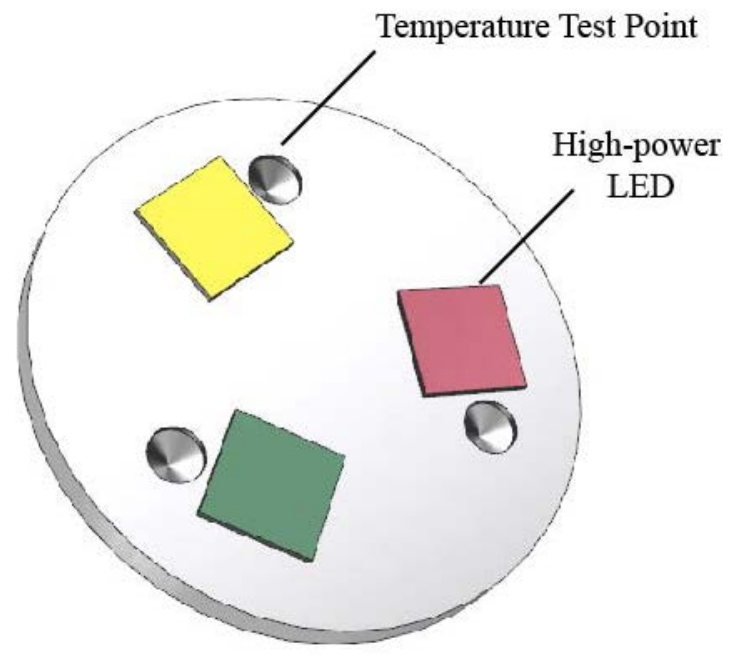

Figure 1. Proposed multi-wavelength light source with individual temperature test point for each LED

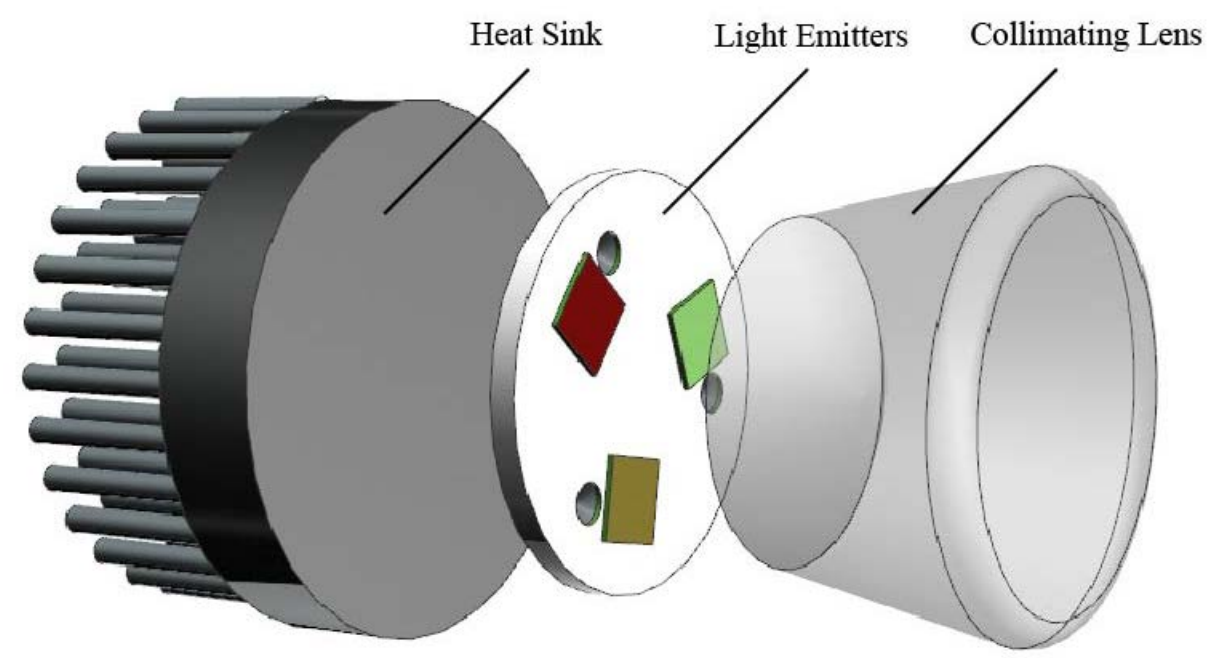

Figure 2. Compact light source assembly

LEDs were attached to an aluminium substrate with high thermal conductivity to divert excessive heat generated by semiconductors with forward currents up to $600 \mathrm{~mA}$. A finned heat sink was bonded to the substrate using a conductive thermal compound to passively cool the light source and keep the semiconductor junction temperature within the recommended range (Figure 2). Thermal management was found to be extremely critical for iPPG performance and reliability, because an increase in the junction temperature had an adverse effect on output optical power and the dominant wavelength and chromaticity shift for monochromatic and white LEDs ${ }^{[11], \text { [12] }}$. The diode forward voltage method was implemented to assess the junction temperature ${ }^{[13]}$. Forward voltage and current through each LED were measured using a dedicated power monitor IC (INA219, Texas Instruments, USA). Surface temperature at test points was recorded by a digital temperature converter (MAX31855, Maxim Integrated, USA) and a thermocouple mounted 
close to LEDs on the metal substrate. These readings were compared with a look-up table provided by the LED manufacturer to estimate junction temperature.

The thermal management system was automated using a MCU (SAM3X8E, Atmel, USA) and set to take measurements when LEDs were active. The recordings were averaged over a 5 second interval to smooth any transient behaviour. In the event of detected wavelength shift or a temperature increase, the forward current through the light source was gradually reduced in steps until the thermal management readings were within a predefined threshold.

LEDs selected for the light source were manufactured with a dome micro-lens to provide 120-degree field of view (FOV). An additional 25-degree FOV collimating lens was placed in from of the light source to collect and concentrate available flux on the region of interest.

\subsection{Camera selection}

Since the slowly varying pulsatile blood component corresponds to only $0.1-1.5 \%$ of all backscattered light ${ }^{[14]}$, it is important to accurately detect these weak pulsations and differentiate them from non-biological noise sources, e.g. micro-motion, ambient light flicker or instrumentation noise. One attainable solution is to employ a photosensor with a high dynamic range, usually 10-bit and higher. In contrast to a widely available 8-bit sensor, which can resolve $2^{8}=256$ light intensity levels, a 10-bit or 12-bit sensor can characterise up to 1024 or 4096 intensity levels respectively. Hence, a high available dynamic range allows detecting weak pulsations of the microvascular tissue bed with greater detail.

In order to investigate the influence of dynamic range on the shape and detail clarity of a PPG waveform, image frames encoded in low and high-bit scale were examined. A 16-bit sCMOS monochromatic sensor (Orca Flash 4.0, Hamamatsu Photonics, Japan) with 2048x2048 effective pixels with 6.5 um cell size was employed in this study. Once the frames were recorded, a down-conversion approach was adopted to reduce the dynamic range down to 8-bit to emulate a low-bit resolution recording process. This scenario was used to obtain two sets of images with the same composition and configuration yet different dynamic range at 16 and 8-bits. The following signal post-processing method was identical for both sets.

This iPPG system was designed to image a $10 \times 10 \mathrm{~cm}$ tissue area. Distances of $0.5-1.5 \mathrm{~m}$ away from the tissue sample were used to simulate various clinical application scenarios. Prime lenses with focus distance of 50 and $85 \mathrm{~mm}$ (Planar $\mathrm{T}^{*}$, Zeiss, Germany) with extended spectrum range were selected to achieve high optical resolution.

The camera was set from a personal computer workstation via a Camera Link interface board using HCImage software (Hamamatsu Photonics, Japan). A control circuit based on a MCU (SAM3X8E, Atmel, USA) was connected to both camera and the light source to temporarily modulate light and control equipment synchronisation. Once triggered by the MCU, camera read out a predefined number of images and transferred them to a PC via a Camera Link frame grabber (FireBird, Active Silicon, UK).

\subsection{Signal processing}

Matlab (MathWorks, USA) software was used to create custom signal processing algorithm. Captured frames were sorted into $\mathrm{N}$ sequences to form 3D matrices of a Rows*Columns*Frame-number type, where $\mathrm{N}$ is the number of individual LED wavelengths used during the experiment. For a mono-wavelength acquisition a single LED was use, while multi-wavelength experiment included 2 or 3 LEDs. A region of interest (ROI) was selected on the first frame and the average of all the pixel values within this region was calculated. iPPG signal was derived from these averaged values across a time sequence of frames. This process yielded multiplexed iPPG signals acquired simultaneously at multiple wavelengths.

$6^{\text {th }}$ order polynomial fit was applied to iPPG signals to determine a quasi-static DC offset and distinguish the low amplitude AC component associated with blood volume changes. A $5^{\text {th }}$ order Butterworth bandpass filter with cutoffs at 0.5 and $4 \mathrm{~Hz}$ was chosen for its flat frequency response to further attenuate noise and fast transitions. The result was an iPPG AC signal describing blood pulsation situation in a small ROI.

In clinical applications it is often desirable to compare a tissue site with underlying pathology to a healthy specimen. Depending on the tissue status and assessment area, iPPG waveform might be corrupted with noise and become undistinguishable in time domain. Frequency domain analysis usually provides no significant improvement since iPPG signal is highly aperiodic resulting in a complex spectrum with both low and high frequency content. In this scenario a method to extract iPPG waveform from a corrupted signal is required. A synchronous detection approach was used to 
distinguish weak signal in a strong random noise background. This method was previously proposed by ${ }^{[15]}$, where a reference function formed from a large area was applied on spatially distributed small ROIs. This paper investigates how the area size used to establish a reference signal influences synchronous detection on various cites of arbitrary size.

Synchronous detection could be briefly described as a signal processing technique were a reference signal with known frequency and phase is applied on a noisy signal to extract even small details with high efficiency. The method is relatively straightforward and requires that the signal of interest should be multiplied by the reference signal and integrated over a finite period. Details that are not synchronized with the reference (not in phase), such as noise, are canceled out. This yields a relative phase and amplitude between two signals, which could be further used for assessing blood perfusion in various regions of interest.

\subsection{Experimental protocol}

Recordings were taken from 3 healthy subjects with no known cardiovascular issues according to the ethical committee approval form Loughborough University. The image sequences were taken in a temperature-stable (23-25 $\left.{ }^{\circ} \mathrm{C}\right)$ dark room environment to avoid uncontrolled influence of ambient and artificial light. Subjects were asked to rest their palms on a soft cushion to minimise motion artefacts. Distances and prime camera lenses were chosen to capture $10 x 10 \mathrm{~cm}$ of palm area on 1000x1000 pixel images within 0.5-1.5 m from the tissue surface. Optical power meter and spectrometer probes were located near areas under examination facing the light source in such manner to avoid shading and interfering with the region of interest. The light source was placed $0.7 \mathrm{~m}$ away from the tissue surface at right angle. The camera was configured to capture 25 frames/second per wavelength with 10-15 ms exposure for 10 seconds. Depending on how many wavelengths were selected for simultaneous image acquisition, the camera frame rate ranged 25-75 frames/seconds. Light source was set to pulse each LED for 10-15 ms. Only one LED was active at any given time, followed by another LED in a continuous loop. Forward current was set individually for each wavelength LED in the range 400-600 $\mathrm{mA}$ to achieve highest dynamic range without sensor being saturated, which was verified using a histogram in HCImage software (Hamamatsu Photonics, Japan).

\section{RESULTS}

\subsection{Influence of camera bit depth}

A down-sampling conversion was applied on 16-bit images to obtain 8-bit images as described previously. As a result, the available dynamic range was reduced from maximum 65,536 to 256. Figure 3 illustrates a signal formed from a 20x20 pixel area and processed using de-trending and filtering. Further analysis by plotting both signals against each other showed no significant influence of the bit-depth on final results. The shape of raw de-trended signals differed slightly around systolic peaks and diastolic troughs, while filtered signals were undistinguishable. The down-conversion of original images indicated a negligible loss in pulse-waveform detail clarity, but reduced the size of transferred and stored images by the factor of two. As a result, iPPG system could potentially achieve higher frame rate since this parameter is proportional to the size of an individual frame. This study, however, did not conclude that a 16-bit camera sensor could be substituted by any 8-bit sensor to achieve the same performance level due to potential differences in sensor architecture and supporting electronics. Further experiment evaluating 8-bit and 16-bit sensor of similar performance and structure should be conducted.

\section{2 iPPG waveform}

A region of approximately $1 \mathrm{~cm}^{2}$ was selected and processed (Figure 4 and Figure 5). High frequency noise and fast transitions could be clearly seen on the raw de-trended curve (a). Bandpass filter compensated majority of fast transitions without over filtering to preserve unique features associated with pulse-wave propagation through vessels. A peak-trough detection algorithm successfully identified systolic (maximum) and diastolic (minimum) values, which defined the length of one cardiac cycle. Figure 6 also shows a dicrotic notch which reflects the closure of the aortic valve and could be further used in assessing the state of the whole cardiovascular system. As the algorithm was automatic, some misdetection had to be manually removed, such as a dicrotic notch at $6.4 \mathrm{~s}$, which should occur after the corresponding systolic phase at time $6.7 \mathrm{~s}$. 

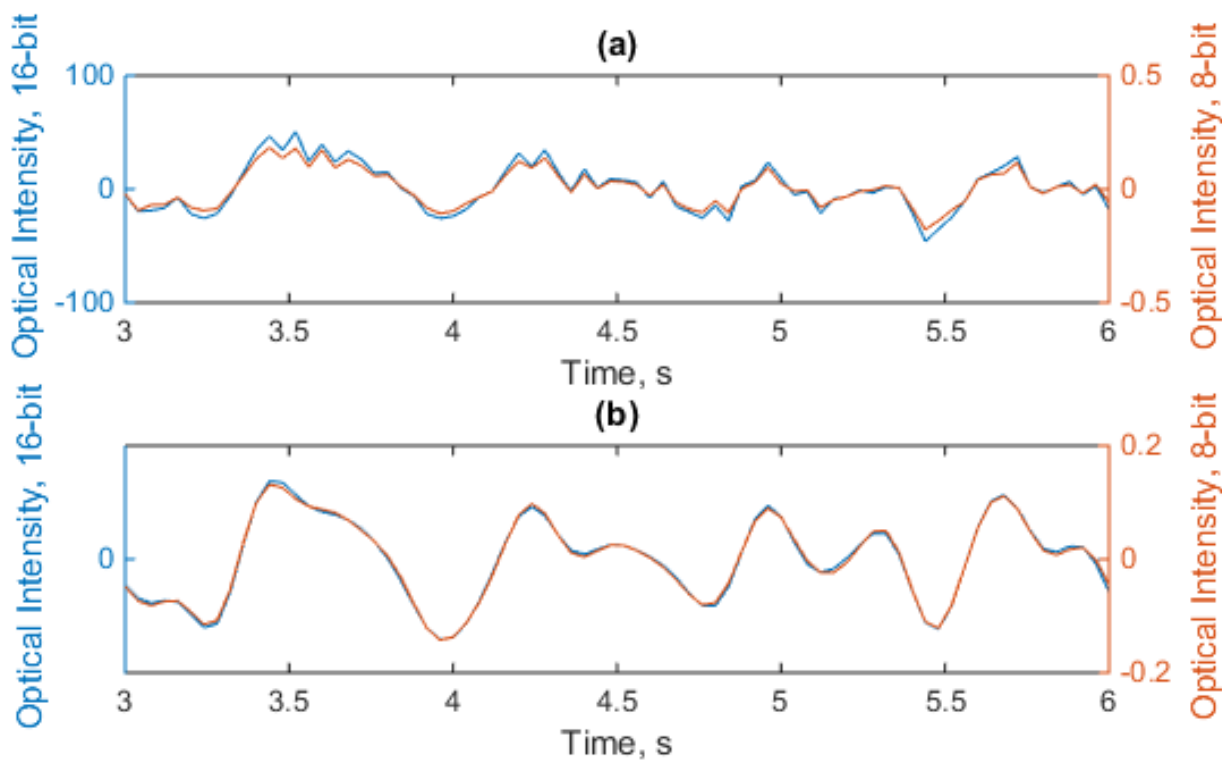

Figure 3. 16-bit and 8-bit iPPG pulse-waveforms derived from a 20x20 pixel area illuminated by amber (590 nm) light. (a)

De-trended raw signals. (b) Bandpass filtered.

Multi-wavelength iPPG system revealed different shape of pulse-time waveforms when the tissue was illuminated by 530 and $590 \mathrm{~nm}$ wavelength light. This indicated not only different penetration depth, but also various microcirculation patterns at different layers in the examined skin tissue. Difference in AC amplitude between two signals, however, could not be compared directly to conclude on stronger or weaker blood pulsations at particular depth since the spectral response of the digital camera is not linear across its working range. If AC amplitudes of a multi-wavelength iPPG system should be compared, the intensity of the backscattered light has to be normalized first by taking into account variations in tissue absorbance and scattering for a particular wavelength, as well as non-linearity in spectral behavior of digital camera and optical components. These problems have to be addressed in further research.

(a)

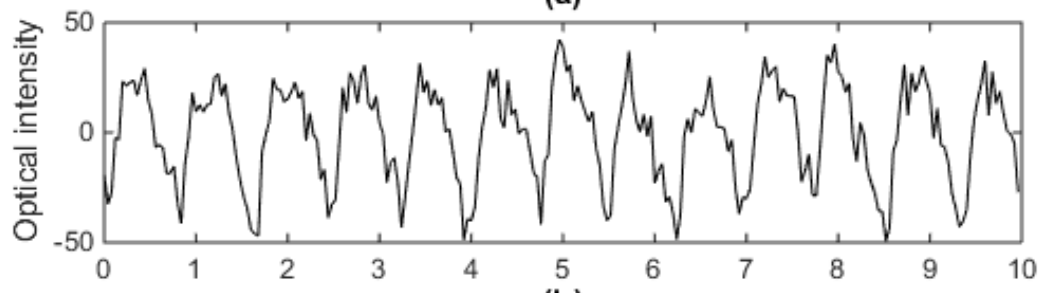

(b)

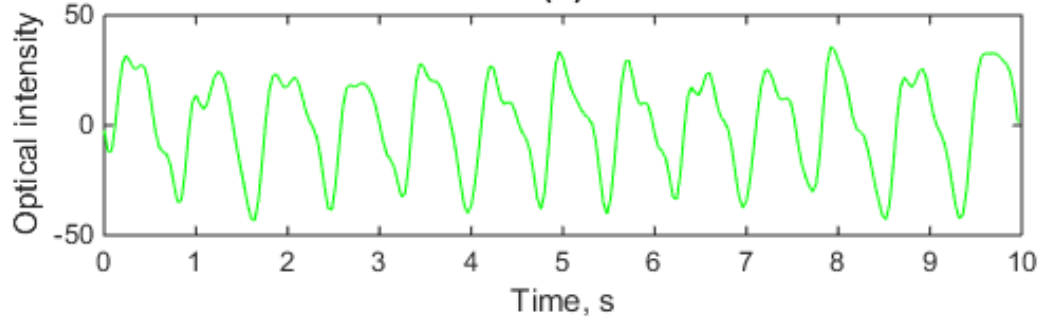

Figure 4. Raw de-trended (a) and filtered (b) iPPG signal under $530 \mathrm{~nm}$ light illumination 
(a)

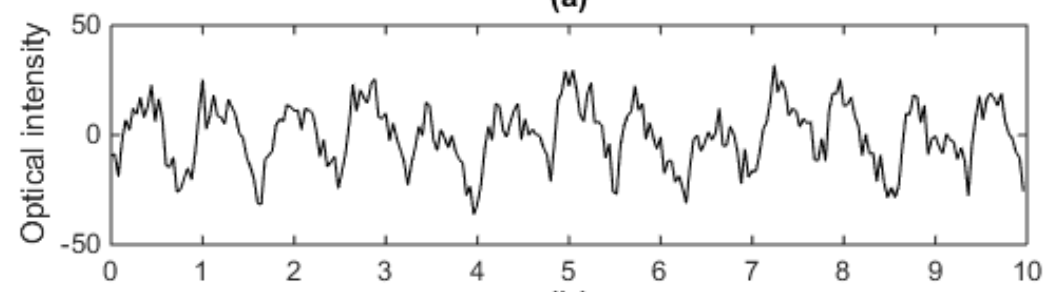

(b)

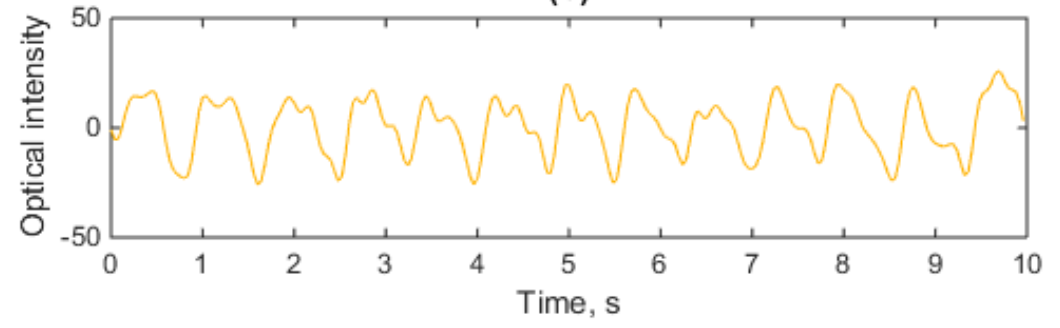

Figure 5. Raw de-trended (a) and filtered (b) iPPG signal under $590 \mathrm{~nm}$ light illumination
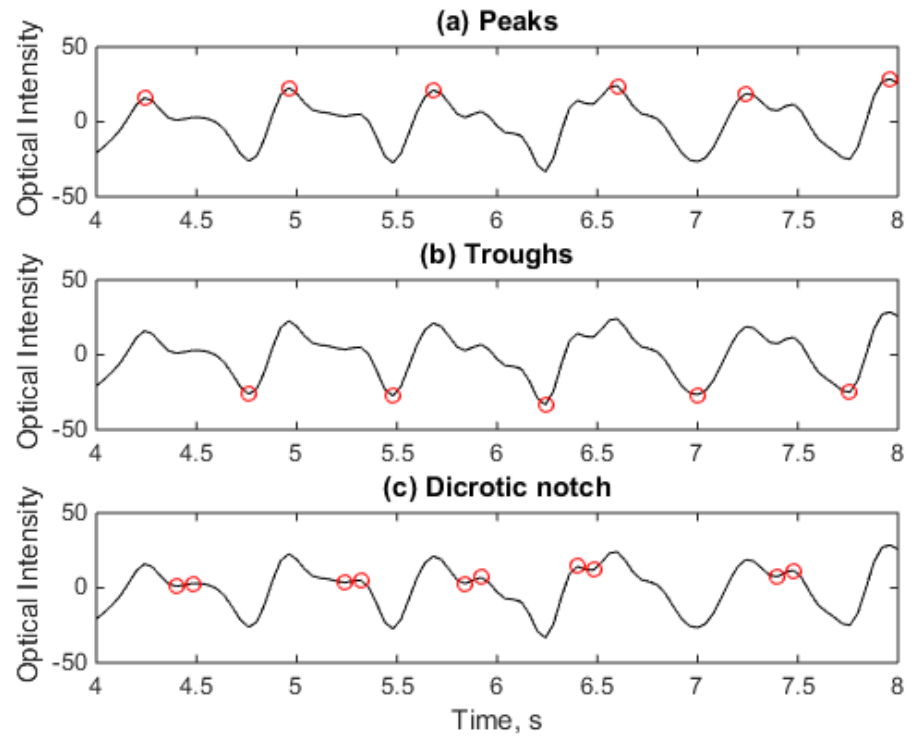

Figure 6. Automatic peak-trough finder algorithm applied to processed pulse-wave. (a) Peaks are associated with systolic phase of a cardiac cycle. (b) Troughs are associated with diastolic phase. (c) Dicrotic notch is associated with a sudden drop in pressure after systolic contraction. One misdetection occurred at 6.4s, which was manually excluded from data set.

\subsection{Synchronous detection}

Once iPPG signal from a selected ROI was filtered and split into cardiac cycles, a reference signal was formed for each of those cycles. Formation started with calculating peak-to-trough amplitude and DC offset followed by computing an approximated first harmonic periodic signal to best fit the original (Figure 7). This resulted in a series of reference signals, which was used to extract AC amplitude and relative phase from any other ROIs on the examined tissue.

Another arbitrary region of interest was selected and processed to obtain iPPG waveform. Figure 8 compares this signal (blue curve) with the reference functions calculated earlier. It can be seen that some systolic peaks in this new region occurred earlier compared to the reference, while others were synchronized. This phenomenon is not unusual and could be of great medical value for certain applications, e.g. assessment of blood vessel elasticity and pulse-wave propagation in tissues. 


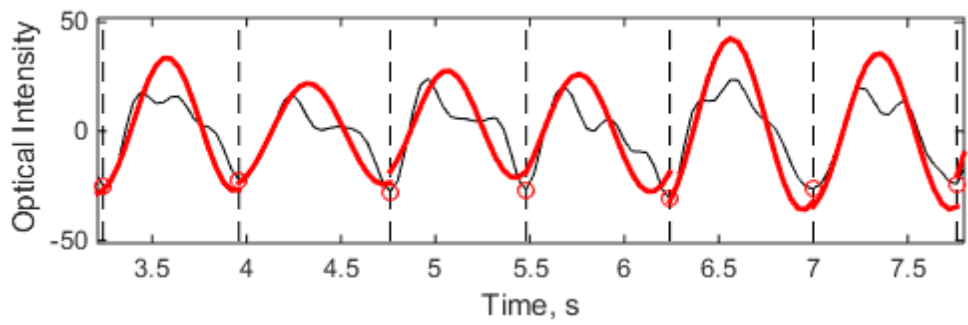

Figure 7. Processed iPPG signal (black) used to obtain reference signal (red). Red circles indicate diastolic trough. Interval between troughs makes one full cardiac cycle.

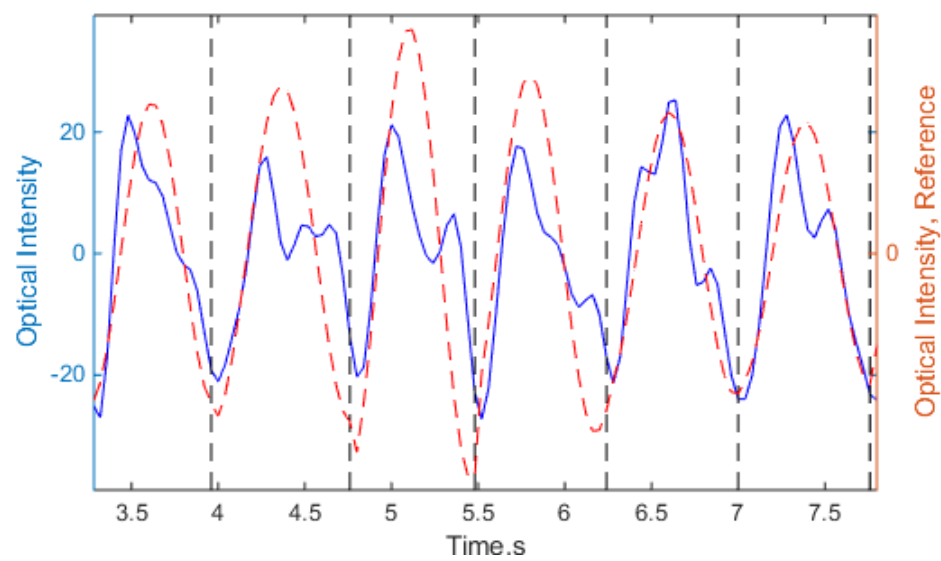

Figure 8. iPPG pulse-waveform obtained from an area under examination (blue). Differences in amplitude and phase are clearly visible when plotted against reference signal (dashed amber)

In order to quantify this relative amplitude and phase, this signal was multiplied by the reference function and integrated over a period of one cycle. The calculated value was a complex number with the real and imaginary parts associated with AC amplitude and phase respectively. Results are illustrated in Figure 9. First and last readings should be ignored because the cardiac cycles where incomplete at the time of recording; future version of this algorithm would include preprocessing to truncate reference signal and include complete cycles only.

It could be seen that within a 10 second recording 12 successful and 2 incomplete cardiac cycles were registered. Most of the diastolic peaks occurred 0.02-0.04 seconds earlier compared to the reference cite. AC amplitude were mostly lower or equal compared to the reference and the difference was within 10 pixel values.

(a)

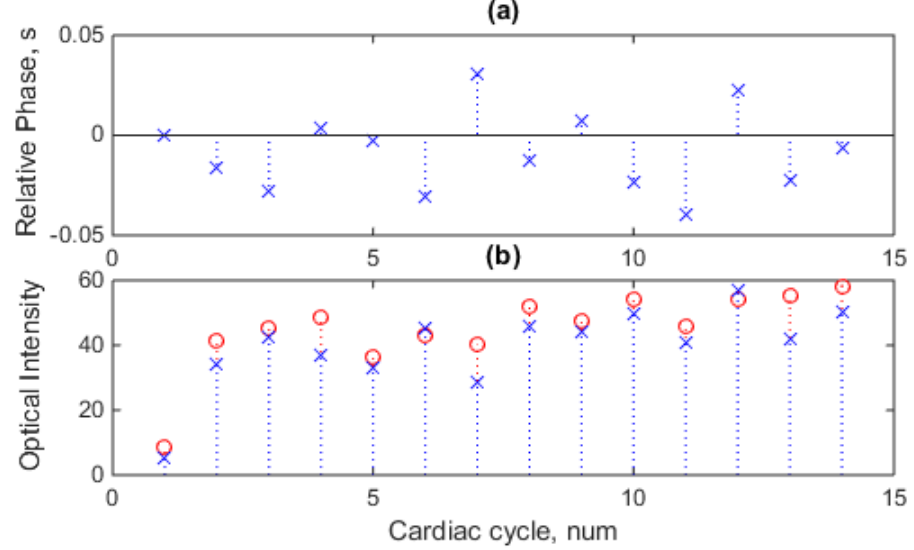

Figure 9. Relative phases difference in seconds (a). Peak-to-trough amplitude of the reference (red) and signal under examination (blue) 


\subsection{Effect of filtering and ROI size}

In the previous section reference function was calculated using de-trended and filtered signal obtained from an area of 1 $\mathrm{cm}^{2}$ ( 100x100 pixels). iPPG waveform from an arbitrary area was also filtered before reference signal was applied to it. Since a digital filter could introduce phase distortion, bandpass ripple and over filtering artefacts, it might be desirable to obtain phase-amplitude maps directly from unprocessed data. Robustness of the synchronous detection method was tested by applying it to unfiltered signal from the same ROI as in Figure 9. There is no significant discrepancy in detected relative phases between unfiltered and processed signals, with only few occasions where value changed sign, e.g. cycle 2 and 9 (Figure 10). AC amplitudes were lower or equal to the reference and followed the same pattern as observed with filtered data in Figure 9.

(a)

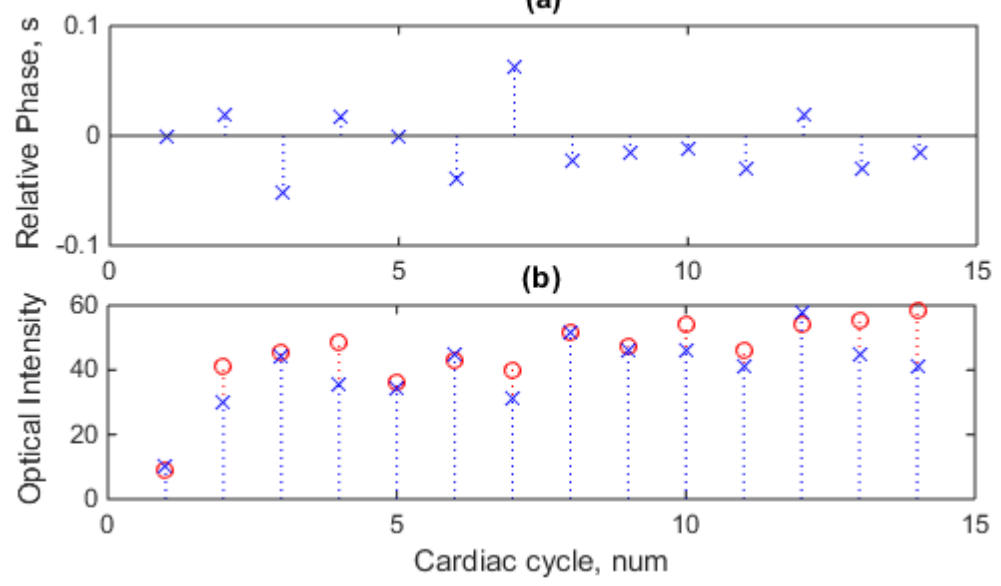

Figure 10. Relative phase difference in seconds (a). Peak-to-trough amplitude of the reference (red) and unprocessed raw signal from the same ROI as in Figure 9

In medical applications assessment of microcirculation within a small site in comparison with healthy tissue is usually desired. However, reduction in ROI size is associated with increased signal-to-noise ratio and higher susceptibility to motion artifacts due to fewer pixels used to find an average value. Experiments involving areas of 100 to $5 \mathrm{~mm}^{2}$ processed with the same reference signal were conducted (Figure 11). Starting with an 100x100 pixels ROI, the examined area was progressively reduced to 20x20 pixels $\left(\sim 5 \mathrm{~mm}^{2}\right)$ in steps of 10 pixels. In situations where peaks and troughs were hardly distinguishable and some cycles contained multiple systolic peaks, the synchronous detection method extracted meaningful data.

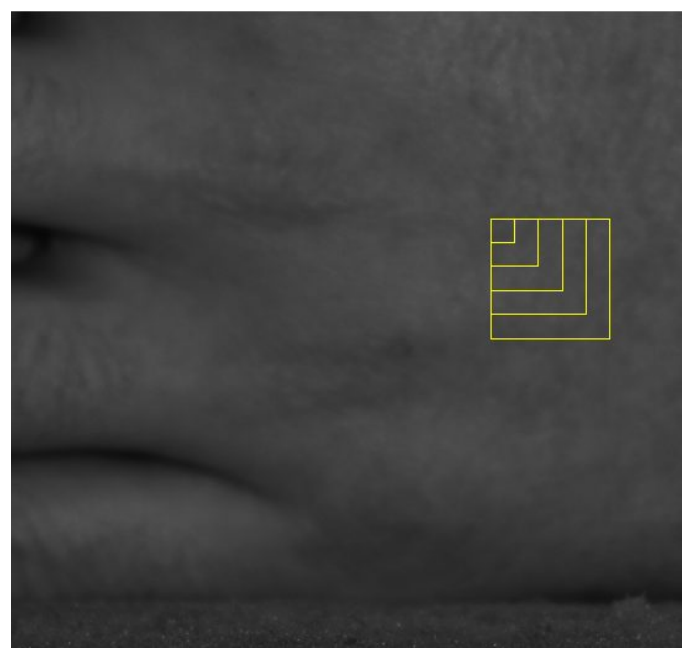

Figure 11. ROI area used to extract iPPG signals from a palm. ROI was progressively decreased from 100x100 to 20x20 pixel site, which was an equivalent of 100 to $5 \mathrm{~mm}^{2}$. 
Raw data from a 20x20 pixel area is shown in Figure 12 where cardiac features, such as peaks, troughs and dicrotic notches were hidden behind strong noise background. Application of sychronous detection allowed the amplitude and relative phases to be succesfully extracted (Figure 13).

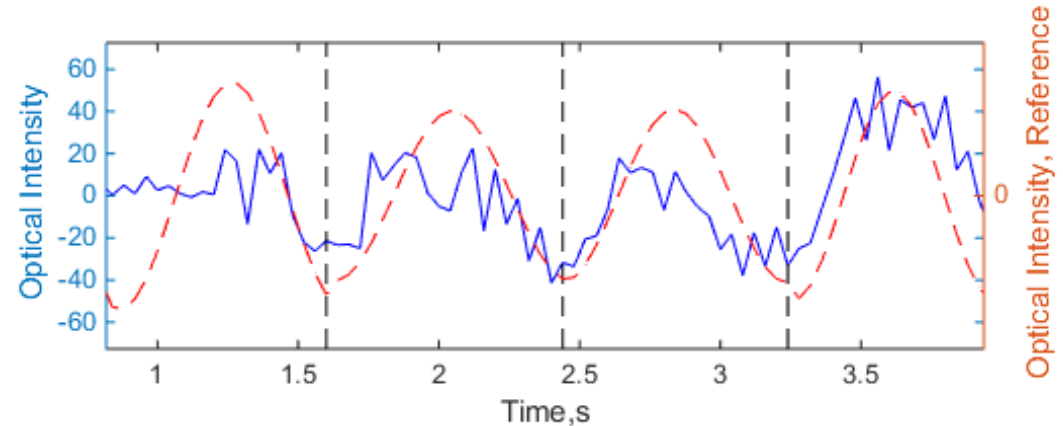

Figure 12. Reference signal (amber) used to extract phase and amplitude from a 20x20 pixels region (blue).

(a)
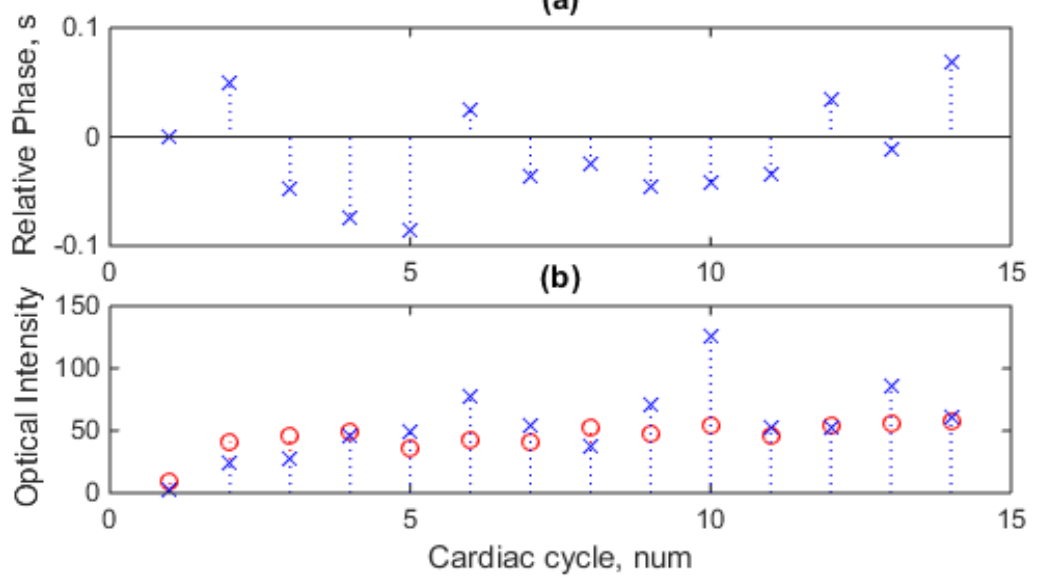

Figure 13. Relative phase difference in seconds (a). Peak-to-trough amplitude of the reference (red) and unprocessed raw signal from Figure 12.

\section{CONCLUSION}

This study demonstrated a novel multi-wavelength light source for iPPG medical applications based on narrowbandwidth high power LEDs. This approach allowed not only simultaneous assessment at various tissue depths, but also provided a simple and cost-effective solution to generate high intensity optical flux of uniform distribution at distances greater that $0.5 \mathrm{~m}$ from tissue surface. A single light emitter per wavelength ensured that the spectral parameters could be easily verified and maintained by monitoring its optical power emission and spectral behavior. A thermal management system was implemented to estimate LED junction temperature and reduce forward current to avoid thermal wavelength shift. A small drift of $6 \mathrm{~nm}$ was registered after $15 \mathrm{~min}$ warm up until the temperature stabilized around $85{ }^{\circ} \mathrm{C}$. This allowed precise control and results repeatability between consecutive measurements in medical applications where exact setup is required, e.g. assessment of wood healing over a span of few days.

Influence of image bit depth on iPPG pulse-waveform detail clarity was assessed. 8-bit images obtained from 16-bit originals to emulate a low bit depth consumer-grade sensor showed no major discrepancy compared to higher bit depth frames. Reduction of the image size by the factor of two might have no effect of iPPG signal, but would significantly reduce storage requirements and potentially increase camera output frame rate. However, no experiment comparing a standalone high and low bit depth sensor was conducted, which would be undertaken in further research.

Synchronous detection method to extract amplitude and relative phase of blood pulse-waves in the region of interest using a reference signal showed promising results. A reference signal derived from an area of $1 \mathrm{~cm}^{2}$ was successfully 
applied to obtain blood perfusion parameters from site up to $5 \mathrm{~mm}^{2}$. The method was proved to be robust when applied to both filtered and unprocessed raw iPPG signals. The results thereby offer further support for the applicability of iPPG to detect and define pulsatile blood microcirculation in tissues and constitute a further step towards noncontact blood perfusion mapping.

\section{REFERENCES}

[1] Texas Heart Institute, "What is a Perfusionist?” <http://www.texasheart.org/HIC/Topics/FAQ/wiperfusion.cfm>

[2] Allen, J., "Photoplethysmography and its application in clinical," Physiol. Meas., vol. 28, no. 3, p. R1, (2007).

[3] Huelsbusch, M. and Blazek,V., "Contactless mapping of rhythmical phenomena in tissue perfusion using PPGI,” Proc. SPIE 4683, 110-117 (2002).

[4] Sun, Y., Papin, C., Azorin-Peris, V., Kalawsky, R., Greenwald, S. and Hu, S., "Use of ambient light in remote photoplethysmographic systems: comparison between a high-performance camera and a low-cost webcam,” J. Biomed. Opt., vol. 17, no. 3, pp. 0370051-03700510 (2012).

[5] Zheng, J., Hu, S., Azorin-Peris, V., Echiadis, A., Chouliaras, V. and Summers, R., "Remote simultaneous dual wavelength imaging photoplethysmography: a further step towards 3-D mapping of skin blood microcirculation,” Proc. SPIE 6850, 68500S-68500S-8 (2008).

[6] Bashkatov, A. N., Genina, E. A., Kozintseva, M. D., Kochubei, V. I., Gorodkov, S. Y. and Tuchin, V. V., "Optical properties of peritoneal biological tissues in the spectral range of 350-2500 nm," Opt. Spectrosc., vol. 120, no. 1, 1-8 (2016).

[7] Fodor, L., Ullmann, Y. and Elman, M. ,"Light Tissue Interactions,” Aesthetic Appl. Intense Pulsed Light, 1133 (2011).

[8] Lister, T., Wright, P. A. and Chappell, P. H., “Optical properties of human skin,” J. Biomed. Opt., vol. 17, no. 9, 0909011-09090115 (2012).

[9] Zheng, J., Hu, S., Echiadis, A. S., Azorin-Peris, V., Shi, P. and Chouliaras, V., “A remote approach to measure blood perfusion from the human face,” Proc. SPIE 7169, 716917-716917-7 (2009).

[10] Humphreys, K., Ward, T. and Markham, C.,"Noncontact simultaneous dual wavelength photoplethysmography: A further step toward noncontact pulse oximetry,” Rev. Sci. Instrum., vol. 78, no. 4, 044304 (2007).

[11] Chhajed, S., Xi, Y., Gessmann, Th., Xi, J. and Shah, J.M.,“Junction temperature in light-emitting diodes assessed by different methods.” <http://www.kaelectronics.com/images/pdf/Junction_Temperature _LED_Tempco.pdf $>$.

[12] Osram Opto Semiconductor, “Thermal Characteristics of LEDs.” <http://ledlight.osram-os.com/wpcontent/uploads/2013/01/Osram-Os_Led-Fundamentals_Thermal-Characteristics-of-LEDs_v2_08-1611_SCRIPT.pdf $>$.

[13] Zabiliute, A., “Temperature characteristics of LEDs.” <http://web.vu.lt/tmi/a.zabiliute/wpcontent/uploads/sites/2/2013/11/LED_Temperature_characterstics.pdf $>$.

[14] Cui, W., Ostrander, L. E. and Lee, B. Y., "In vivo reflectance of blood and tissue as a function of light wavelength,” IEEE Trans. Biomed. Eng., vol. 37, no. 6, 632-639 (1990).

[15] Kamshilin, A. A., Miridonov, S., Teplov, V., Saarenheimo, R. and Nippolainen, E., "Photoplethysmographic imaging of high spatial resolution,” Biomed. Opt. Express, vol. 2, no. 4, 996 (2011). 